\title{
L'IMPLANTATION DES ÉDIFICES CULTUELS DES TÉMOINS DE JÉHOVAH EN FRANCE
}

\section{Régis DERICQUEBOURG}

Le jéhovisme, issu du Mouvement des Étudiants de la Bible fondé aux États-Unis par Charles Taze Russell (1852-1916) fait aujourd'hui partie du paysage religieux de la France (Introvigne, 1990 ; Blandre, 1991 ; Barbey, 2003). Chaque jour des Témoins de Jéhovah en prédication sonnent aux portes. Ils laissent à ceux qui leur prêtent attention deux revues : La tour de garde et Réveillez-vous ; ils leur proposent l'étude gratuite de leur enseignement et ils les invitent à écouter le « discours biblique » du dimanche après-midi dans leurs lieux de culte.

Le premier proclamateur du russellisme en Suisse, en France et en Belgique a été Adolf Erwin Weber, né le 19 juin 1863 à Brüttelen et mort le 4 février 1948 à la Chaux-de-Fond. Dans sa jeunesse, il s'était rendu aux États-Unis où, par hasard, il était devenu le jardinier de C. T. Russell. Fidèle des conférences de son patron, il avait été convaincu par son interprétation des Écritures, et s'était fait baptiser en 1890. Après son retour en Suisse vers 1895, Weber travaille comme jardinier et comme forestier tout en faisant du prosélytisme. Il prospecte dans le canton de Vaud en utilisant sa propre traduction du premier volume des Études dans les écritures de Russell et du premier numéro du périodique des Étudiants de la Bible : Le phare de Sion (octobre 1903). Sa stratégie consiste à passer des petites annonces dans les journaux et dans les périodiques locaux pour proposer les Écrits de Russell. Chaque hiver, il se rend en France, en Belgique et en Italie pour faire de la prédication. En 1904, il vient en France dans la région du Doubs. Il se rend ensuite à Charleroi et à Ampsin (Belgique) où il fonde les premiers groupes d'Étudiants de la Bible. Avec quelques proclamateurs belges 1 , il prospecte dans le bassin minier du nord de la France, distribuant des tracts à la sortie des temples baptistes. Sa prédication porte ses fruits puisqu'en 1906 quelques baptistes quittent leur congrégation pour former à Denain (Nord) un petit groupe d'Étudiants de la Bible qui se réunit chez les uns et les autres pour étudier les Écrits de Russell. De 1906 à 1909, le mouvement se propage dans le Bassin minier : un groupe important (ecclesia) se constitue à Lens puis d'autres à Hénin-Liétard, Auchel, Flinesles-Râches, Sin-le-Noble. Un autre fondé à Roubaix. Le mouvement recrute essentiellement des mineurs et des ouvriers. Entre 1914 et 1918, d'autres groupes se forment à Paris, Le Havre, Sainte-Suzanne (Mayenne), Oyonnax, Rodez, Montceau-les-Mines, Bruay-en-Artois (Pas-de-Calais). Le mouvement des Étudiants de la Bible démarre cependant lentement en France par comparaison avec les pays anglophones et scandinaves. Russell attribuait ce fait à l'influence " néfaste » des catholiques et il n'investissait pas beaucoup dans le prosélytisme en France. Mais le russellisme s'est tout de même développé dans le Nord-Pas-de-Calais au point que sur les cinq périples que Russell effectua en France, deux le firent passer par cette région, dans des petites villes comme Denain et Lens. La commémoration de la Cène de 1914 réunit 48 personnes. À partir de 1920, le recrutement s’intensifie dans la région minière grâce à l'apport d'émigrés polonais. Certains de ces émigrants venus d'Allemagne (les Polono-Westphaliens) avaient déjà été contactés par des Étudiants de la Bible en Westphalie. On a qualifié l’activité des Étudiants de la Bible de « religion des Polonais ». Les Zloty Wiek, 
titre leur revue (l'Âge d'or) en langue polonaise (Dericquebourg, 1977). En 1917, un an après la mort de C. T. Russel (1916), le mouvement des Étudiants de la Bible s'est scindé en deux branches à l'issue d'un conflit entre les prétendants à la succession du prophète fondateur. L'une, plus active, a pris en 1931, le nom de « Témoins de Jéhovah ». Son véritable démarrage en France s'effectue dans les années 1950. Depuis cette date, le nombre de fidèles est passé de 4600 à 133000 en 2001.

\begin{tabular}{|c|c|c|c|c|c|c|}
\hline $\begin{array}{c}\text { Les Témoins de Jéhovah en } \\
\text { France }\end{array}$ & \multicolumn{2}{|l|}{} \\
\hline & 1951 & 1961 & 1971 & 1981 & 1991 & 2001 \\
\hline Témoins actifs & 4600 & 16200 & 44500 & 76200 & 133100 & 133000 \\
\hline Assistance mémorial & 9800 & 24800 & 80300 & 157200 & 267700 & 247700 \\
\hline
\end{tabular}

En 1922, la plus ancienne Salle du Royaume de France fut construite à Denain. On comptait alors 300 fidèles en France. Ils sont 1040 en 1939. Leurs activités furent interdites pendant la seconde guerre mondiale (association étrangère) mais en 1947, quand leur interdiction fut levée, 4000 Témoins assistèrent à l'assemblée de District de Douai (rassemblement régional) et ils furent 10500 au rassemblement national de Paris en 1950. En 1963, ils étaient 23000 en France puis 29000 en 1969. Pendant toute la période 1922-1975, les Témoins ont consolidé leurs positions en étoffant le Bassin minier et en réussissant leur implantation dans la région textile de Lille Roubaix -Tourcoing puis dans les zones industrielles du Littoral (DunkerqueOutreau). En 1975, sur les 65000 Témoins de France, 15000 habitaient la région Nord-Pasde-Calais. Les régions rurales ont résisté à leur avancée. Le Président de la Watchtower Societyㄹ de l'époque (Rutherford), visitant une congrégation de Bruay-en-Artois en 1924, rend hommage au zèle des franco-polonais dans l'Annuaire des Témoins de 1929. Il signale qu'ils parcourent la région faisant parfois 100 kilomètres à bicyclette pour contacter d'autres émigrés polonais et qu'en 1929, 332 émigrés polonais ont pris le baptême. Puis le jéhovisme a touché les émigrés italiens, espagnols et portugais. Récemment, une congrégation dite « orientale » (français d'origine maghrébine) s'est ouverte à Roubaix. On trouve d'autres congrégations de ce type en France, mais les conversions des musulmans au jéhovisme restent peu importantes. Smaïl Medhaoui qui a enquêté sur ce phénomène à Roubaix a constaté que les Témoins recrutent principalement des personnes originaires de la Kabylie (Médhaoui, 1999). 


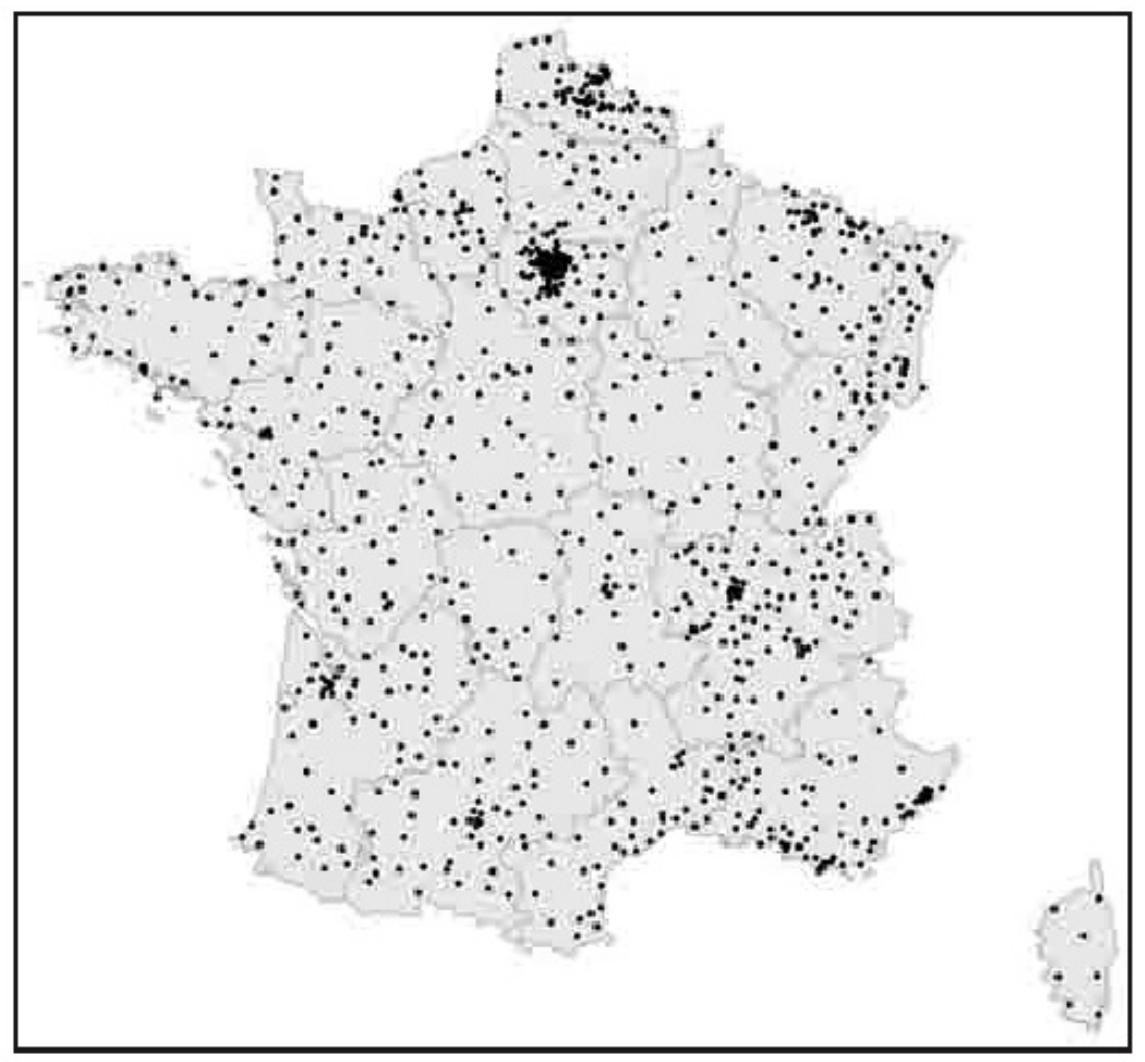

Carte des salles du Royaume sur le territoire français métropolitain.

Cartes des salles du Royaume sur le territoire français métropolitain

Dans d'autres régions, ils recrutent, comme les assemblées évangéliques, des Français d'origine africaine, des Réunionnais et des Antillais. Comme l'a montré la littérature consacrée aux mouvements millénaristes (Kauffman, 1964 ; Talmon, 1966 ; Séguy, 1966), les Témoins ont longtemps recruté et recrutent encore parmi les populations émigrées et défavorisées, les deux se confondant souvent. Dans son enquête réalisée (entre 1965 et 1969), Quirinus J. Münter (1977) relève que 40 «proclamateurs » sur 86 pensent que les travailleurs manuels sont plus sensibles à leur message.

On constate cependant depuis une vingtaine d'années des conversions au jéhovisme de personnes situées dans la Lower-Middle-Class et dans la Middle-Middle-Class. L'un des indicateurs du niveau des recrues est le niveau d'études des Témoins masculins : $18 \%$ ont le niveau du bac, $15 \%$ ont le niveau d'instruction bac $+2,3 \%$ ont atteint le niveau bac +5 , (Sofres, 1998). Les Témoins enregistrent une légère décrue depuis quelques années, mais un responsable de l'Association culturelle des Témoins de Jéhovah nous a dit que les statistiques de son mouvement sont plutôt en dents de scie.

\section{État de l'implantation des salles du Royaume}

Des Témoins de Jéhovah qui se réunissent pour célébrer le culte et pour exercer des activités y afférant (formations du proclamateur, étude biblique) forment une congrégation. À l'exception de communautés naissantes qui se réunissent chez un particulier, la pratique religieuse a lieu dans une Salle du Royaume dont les fidèles ne sont pas nécessairement propriétaires $\underline{3}$. 
Ce peut être un local loué et aménagé. Ils peuvent partager avec d'autres congrégations, dont l'une est propriétaire ou locataire, un édifice cultuel. Un responsable du jéhovisme français nous a signalé l'existence d'une salle située en Île-de-France servant à six congrégations. La carte des salles du Royaume ne reflète donc pas le nombre de congrégations. Ainsi au 1er janvier 2003, on comptait 1495 congrégations jéhovistes en France métropolitaine mais l’inventaire que nous a livré le Bethel (Quartier général) de Louviers comprend 918 salles. Pour 772 d'entre elles, la date de construction est connue. En sondant quelques-unes des salles non datées, nous avons appris qu'il s'agissait de bâtiments loués et transformés en lieu de culte.

D'autre part, en consultant la liste, nous nous sommes aperçus que des salles construites à une certaine date ne faisaient parfois que remplacer une salle édifiée antérieurement. Par exemple, celle de Wattrelos (Nord) datée de 1992 remplace une salle construite en 1963, puis surélevée en 1967 pour accueillir quatre congrégations. À Denain (Nord), on trouve l'une des plus anciennes salles du royaume (1922). Celle-ci a été remplacée par une nouvelle salle bâtie en 1987.

Ces cas de reconstruction sont peu nombreux.

La construction d'une salle ne suit pas immédiatement la création d'une congrégation. Celleci doit atteindre un certain niveau de développement et de stabilité (de nouveaux baptisés peuvent remplacer les personnes démissionnaires ou quittant la localité et les personnes décédées). L’installation d'une salle louée peut être lente. Anne-Sophie Labbé (1999) a relaté, à partir d'une recherche locale, comment la congrégation d'Avesnes-sur-Helpe (Nord) s'est constituée à partir de la prédication d'une personne qui a réuni les convertis à son domicile puis dans une salle louée à la Mairie puis dans une autre salle. Ce processus d'installation a commencé peu avant la seconde guerre mondiale pour s’achever en 1984. La date de construction d'une salle indique probablement qu'une congrégation a atteint une force qui lui permet d'envisager un avenir. Des congrégations naissantes peuvent disparaître comme à Montreuil (Pas-de-Calais). Il arrive parfois qu'un lieu de culte soit bâti puis vendu quand la congrégation ne parvient pas à durer.

Pour construire une salle du Royaume, l'association locale des Témoins de Jéhovah ou une association constituée dans ce but lance un emprunt sans intérêt avec un échéancier de remboursement auprès des fidèles ou auprès de l'Association pour la construction des édifices du culte $\underline{4}$. Elle peut également solliciter une banque. L'Association culturelle des Témoins de Jéhovah de France n’est pas propriétaire de l'édifice culturel.

Essentiellement centré sur le pays minier du Nord-Pas-de-Calais au départ, le mouvement jéhoviste s'est propagé peu à peu dans le pays. Anne Sophie Labbé (1999) a montré à l'aide d'un exemple précis que les Témoins ont progressé de proche en proche dans la région de Valenciennes (Nord). Comme le montre notre relevé du nombre de salles du Royaume par départements, en 2003, les Témoins de Jéhovah sont présents dans toute la France métropolitaine. Ils sont également présents dans les DOM-TOM. Toutefois, la carte montre que la répartition des salles de culte jéhoviste n’est pas homogène. Nous trouvons une forte densité d'édifices cultuels dans les zones urbaines : Lille-Roubaix-Tourcoing, Île-de-France, Metz, Strasbourg, Mulhouse, Lyon et sa banlieue, Clermont-Ferrand, Marseille, Nice, Toulouse, région bordelaise. Dans les zones rurales, les salles sont plus dispersées. Le phénomène est visible dans le Nord-Pas-de-Calais : la Flandre Intérieure, l’Audomarois, l’Artois, le Ternois, la Plaine du Boulonnais n'ont pratiquement pas de salles.

\section{Période Nombre de constructions \% du nombre de salles Recrutements}




\begin{tabular}{|c|c|c|c|}
\hline $1937-1960$ & 9 & 1,16 & $?$ \\
\hline $1961-70$ & 27 & 3,49 & 11600 \\
\hline $1971-80$ & 92 & 11,91 & 31700 \\
\hline $1981-90$ & 410 & 53,10 & 56900 \\
\hline $1991-2000$ & 226 & 29,27 & 0 \\
\hline $2001-2003$ & 8 & 1,03 & -17586 \\
\hline Total & 772 & 99,96 & \\
\hline
\end{tabular}

\section{Dynamique de construction des salles}

Les constructions de salles ont-elles accompagné l'évolution du nombre de Témoins en France ? Pour répondre à cette question, nous avons établi un relevé des constructions de salles du Royaume dans les décennies précédentes. Le tableau suivant montre une grande variation dans la construction des salles selon les décennies. On constate que 53,10\% des salles ont été construites entre 1981 et 1990 et que 29,27 \% des salles l'ont été entre 19912000. En tout 82,3 \% des lieux de culte jéhoviste ont été édifiés entre 1981 et 2000. Ces deux décennies n’ont pas été marquées par une annonce datée du retour du Christ, alors qu'une attente s'était propagée dans le mouvement pour l'année 1975. En revanche, elles correspondent à la montée de la controverse sur les groupes religieux minoritaires. La croissance du jéhovisme a marqué le pas entre 1996 et 2001 (avec un recul de $2 \%$ des effectifs en 2001 par rapport à 2000). Ce phénomène n’est pas une spécificité française ; on le retrouve dans d'autres pays. Les responsables du Jéhovisme français ne s'en inquiètent pas. La stagnation n'est peut-être qu'un phénomène passager. La génération de Témoins convertis des années 1950 qui a nettement fait croître la société de la Tour de Garde en France est peutêtre en train de disparaître. Toutefois, la stagnation ou la légère inversion des statistiques de croissance ne semble pas avoir affecté le dynamisme constructeur des congrégations locales.

La controverse à propos des sectes (1981-2000) a peut-être incité à la construction de salles puisque les congrégations locales ont rencontré plus de difficultés à louer des locaux à des propriétaires rendus méfiants par les mises en garde contre les groupes religieux minoritaires. Les plaintes déposées en justice à propos de refus de location de locaux par divers autres petits mouvements spirituels appuient l’hypothèse.

\section{Salles du Royaume et congrégations}

Les congrégations sont plus nombreuses (1495) que les salles du Royaume bâties par les Témoins (918 maximum). Sur les 22 régions de France, il y a en moyenne 3,49 congrégations pour 100000 habitants et 2,38 salles construites pour 100000 habitants $\underline{5}$. Le ratio construction/congrégation s'élève à 61,19. Un peu plus de la moitié des congrégations ont donc bâti leur édifice cultuel.

\begin{tabular}{|l|l|l|l|}
\hline Départements & & Nombre de salles du Royaume & \\
\hline Ain & 9 & Lozère & 2 \\
\hline Aisne & 8 & Maine-et-Loire & 7 \\
\hline Allier & 7 & Manche & 9 \\
\hline Alpes de Haute-Provence & 5 & Marne & 7 \\
\hline
\end{tabular}




\begin{tabular}{|c|c|c|c|}
\hline Hautes Alpes & & Haute-Marne & 4 \\
\hline Alpes Maritimes & 3 & Mayenne & 3 \\
\hline Ardèche & 7 & Meurthe-et-Moselle & 14 \\
\hline Ardennes & 5 & Meuse & 4 \\
\hline Ariège & 4 & Morbihan & 8 \\
\hline Aube & 5 & Moselle & 21 \\
\hline Aude & 5 & Nièvre & 6 \\
\hline Aveyron & 6 & Nord & 42 \\
\hline Bouches du Rhône & 22 & Oise & 8 \\
\hline Calvados & 13 & Orne & 5 \\
\hline Cantal & 3 & Pas-de-Calais & 30 \\
\hline Charente & 7 & Puy-de-dôme & 111 \\
\hline Charente-Maritime & 12 & Pyrénées-Atlantique & 11 \\
\hline Cher & 6 & Hautes-Pyrénées & 5 \\
\hline Corrèze & 7 & Pyrénées-orientales & 9 \\
\hline Corse & 9 & Bas-Rhin & 15 \\
\hline Côtes d’Or & 7 & Haut-Rhin & 9 \\
\hline Côte d'Armor & 6 & Rhône & 22 \\
\hline Creuse & 9 & Haute-Saône & 5 \\
\hline Dorgogne & 9 & Saône-et-Loire & 11 \\
\hline Doubs & 9 & Sarthe & 9 \\
\hline Drôme & 11 & Savoie & 7 \\
\hline Eure & 10 & Haute-Savoie & 14 \\
\hline Eure-et-Loire & 5 & Paris & 10 \\
\hline Finistère & 14 & Seine-Maritime & 22 \\
\hline Gard & 13 & Seine-et-Marne & 16 \\
\hline Haute-Garonne & 16 & Yvelines & 13 \\
\hline Gers & 6 & Deux-Sèvres & 6 \\
\hline Gironde & 23 & Somme & 9 \\
\hline Hérault & 12 & Tarn & 7 \\
\hline Ille-et-Vilaine & 10 & Tarn-et-Garonne & 3 \\
\hline Indre & 6 & Var & 18 \\
\hline Indre-et-Loire & 9 & Vaucluse & 11 \\
\hline Isère & 23 & Vendée & 9 \\
\hline Jura & 6 & Vienne & 6 \\
\hline Landes & 6 & Haute-Vienne & 4 \\
\hline Loire & 14 & Vosges & 12 \\
\hline Haute-Loire & 14 & Yonne & 7 \\
\hline
\end{tabular}




\begin{tabular}{|l|l|l|l|}
\hline Loire-Atlantique & 15 & Territoire de Belfort & 4 \\
\hline Loiret & 7 & Essonne & 13 \\
\hline Lot & 4 & Hauts de Seine & 10 \\
\hline Lot et Garonne & 6 & Seine-St-Denis & 17 \\
\hline & & Val d'Oise & 11 \\
\hline
\end{tabular}

Quand le nombre de fidèles ou le nombre de congrégations augmente, le nombre de salles bâties croît. La tendance est linéaire. On la trouve donc dans chaque région. Cela peut paraître a priori banal mais ça ne l'est pas. En premier lieu, les Témoins qui attendent la fin du monde peuvent être indifférents à la possession d'un lieu de culte. En effet, la préoccupation première est de se conformer à la volonté de Jéhovah pour gagner le salut et d'avertir l’humanité pour sauver les hommes. Dans le cas présent, les Témoins s’installent durablement dans le monde même si cela est une vanité. Renoncent-ils à une fin du monde très proche comme les Adventistes qui se sont installés pour durer après plusieurs attentes ? Ce serait conforme à l'évolution des mouvements eschatologiques repérée par les sociologues. L'enquête de la Sofres (1998) montre que, bien qu'attendant la fin du monde, les Témoins sont plus actifs que la moyenne des Français dans leur épargne et qu'ils accèdent à la propriété, ce qui au premier abord peut être considéré comme paradoxal mais qui ne l'est peut-être pas. En second lieu, en examinant d'autres groupes religieux, on s'aperçoit que la construction de temples n'est pas nécessairement corrélée à une élévation significative du nombre de fidèles. Par exemple, les Antoinistes renversent le processus. Dès qu'il y a quelques adeptes réguliers, une salle de lecture ou un temple est créé car dans les vues antoinistes, ces lieux de réunion ou de culte vont attirer des personnes. La seule nécessité est d'avoir un desservant pour s'occuper du temple.

Dès lors, les édifices cultuels sont antérieurs à la formation d'une communauté et à la constitution d'un public de sympathisants. On trouve chez les Témoins et les Antoinistes deux logiques foncières différentes. Chez les premiers, la salle du Royaume est la conséquence de la formation d'une communauté, chez les seconds, le temple est un élément de proposition religieuse visible (l’antoinisme ne fait pas de prosélytisme organisé).

\section{Emplacement et architecture des salles du Royaume}

L'emplacement d'une future salle du Royaume est choisi en fonction du prix des terrains et de leur disponibilité. Les parcelles doivent être suffisamment grandes pour aménager une aire de stationnement (le Bethel de Louviers nous a signalé que cela correspond à des règles d'urbanisme). Elles doivent se situer à proximité des lieux de recrutement, c'est-à-dire le plus souvent à la périphérie des villes où on trouve des immeubles à loyer modéré et où on construit des lotissements en accession à la propriété. L’achat du terrain et le montant de la construction incombant aux congrégations locales, les salles sont le plus souvent édifiées dans les zones interstitielles entre les villes là où le foncier est moins cher.

Du point de vue architectural, nous constatons une évolution dans l'aspect des salles. Avant la guerre et dans l'immédiat après guerre, les Témoins ont utilisé des bâtiments préfabriqués. Ils ont réhabilité d'anciens entrepôts ou encore d'anciens ateliers (comme l'ex salle de LilleMoulin). Cette modestie était voulue. Dans un vade mecum jéhoviste (WTBS, 1996) nous lisons le conseil suivant : « La salle du royaume qui est notre lieu de culte, ne devrait pas être un bâtiment prétentieux conçu pour impressionner les voisins. Bien que son architecture puisse varier d'un endroit à un autre, elle doit surtout être fonctionnelle». Mais, peu à peu, ils 
ont construit des salles en « dur » très simples puis ils ont fait un effort architectural et parfois d'embellissement par des plantations.

Le Bethel de Louviers inauguré en 1997 est imposant. Assiste-t-on au même phénomène chez les musulmans de France qui passent du lieu de prière dans des salles prêtées au rez-dechaussée ou dans les sous-sols des grands ensembles aux mosquées qui s’embellissent?

Parfois, et de plus en plus, comme à Saint-Aunes (Hérault), les Témoins font appel à un architecte. L’ouvrage a été remarqué dans la revue : Architecture Méditerranéenne ( ${ }^{\circ}$ 30, 1987, ed. Robert Kahiat) en ces termes «Consulté par les Témoins de Jéhovah, l’architecte a conçu pour leurs rassemblements cet édifice solide ancré sur terre, fermé aux tourbillons du monde temporel et simplement ouvert à la lumière céleste, à ses pulsations secrètes. Il a imaginé et construit un volume monolithique et monochrome, sobre mais imposant. Le déploiement des vigoureux plissements renforce le percement de pénétration, exalte par là même la dimension métaphysique et spirituelle de cet espace de recueillement ».

Cette évolution dans les constructions reflète-t-elle un changement de perspective du jéhovisme ? Ce choix d'une véritable architecture correspond-il à l'élévation du statut social des convertis qui souhaitent pratiquer leur culte dans des salles qui peuvent soutenir la comparaison avec les édifices des Églises établies ? En se dotant de salles plus belles, les Témoins souhaitent-ils se donner une plus grande visibilité sociale ? Il est difficile de répondre avec certitude à ces questions car les dirigeants n'ont pas de politique officielle de construction. Ils peuvent aider leur développement mais c'est la congrégation locale qui est le maître d'œuvre. En ce cas, l'embellissement de leurs édifices cultuels reflèterait la mentalité actuelle des fidèles plus que celle des responsables nationaux.

Il faut mentionner un autre aspect. Depuis une vingtaine d'années les Témoins sont passés maîtres dans l'art de construire une salle du Royaume en trois jours et même en 32 heures (à Hénin-Beaumont, Nord, en 1986, salle de 230 m2) tel un défi. Dans l’Indépendant du Haut Jura du jeudi 20 août 1987, on peut lire sous la plume de Nicole Thomas : « Samedi et dimanche dernier, le petit village de Houtaud, situé à trois kilomètres de Pontarlier fut envahi par près de 2000 Témoins de Jéhovah. Ils s'étaient donné rendez-vous, le temps d'un seul week-end, pour construire une salle de réunion de 300 m2! Les travaux commencés dès sept heures le samedi, étaient terminés le dimanche en début d'après-midi. La première réunion pouvait commencer...». Nous trouvons le même type de reportage dans le Dauphiné Libéré du 27 novembre 2000 (qui souligne que le bâtiment en forme de chalet s'intègre bien au paysage) et dans le Journal de Saône-et-Loire du 15 juin 1992. Au total, un peu plus de 80 salles du Royaume auraient été construites en un week-end.

\section{Les difficultés liées à l’implantation des salles du Royaume}

Jusqu'à l'époque de la controverse sur les sectes, les Témoins de Jéhovah ne rencontraient pas de difficulté pour acquérir des terrains et obtenir des permis de construire. Les difficultés sont apparues avec la publication d'un rapport parlementaire sur les sectes qui contient une liste de mouvements « dangereux » dans laquelle ils figurent. En accordant un permis de construire, un maire risque d'encourir des critiques.

Pour s’en prémunir, il tente de refuser le permis de construire, ce qui doit être juridiquement motivé (circulaire Delevoye-Vivien). Souvent, il utilise le droit de préemption en arguant d'un plan d'occupation des sols. 
Les Témoins ont alors la possibilité de recourir à une juridiction administrative. Cette dernière peut demander à la municipalité de revoir le dossier et de conclure au terme d'un délai fixé. Comme la législation sur les plans d'occupation des sols est précise, les Témoins n’ont pas de difficulté à montrer que le refus du maire n’est pas juridiquement fondé. Les procès se sont multipliés durant ces dernières années.

On en trouve une illustration récente dans le jugement de la Cour Administrative d'appel de Paris du 7 février 2003 ( $N^{\circ}$ 99PA01713-Association culturelle des Témoins de Jéhovah de Paris Austerlitz/Mairie de Paris). Dans ce cas il s’agit d'une affaire débutée en 1996 et qui sept ans après, n’était pas terminée puisque la Mairie de Paris devait prendre une décision avant l'expiration d'un délai de quatre mois à compter de la notification de l'arrêt.

La cour peut tout simplement annuler le refus du permis de construire et les travaux peuvent commencer.

On en trouve un exemple dans un jugement du Tribunal administratif de Versailles du 30 mars 1993 : « l'arrêté susvisé du maire de Villiers-le-Bel en date du 3 juillet 1991 est annulé ». De plus, dans un article : "Le droit de l'urbanisme : une arme contre les sectes », Isabelle Rouvière-Perrier (1994) relève un jugement du tribunal administratif de Rouen du 23 février 1994. Dans celui-ci, le juge a estimé que le droit de préemption invoqué par la mairie de Caudebec-les-Elbeuf après avoir accordé aux Témoins un premier permis de construire, avait été destiné à empêcher la construction d'une salle du Royaume à la suite de protestations locales. Le juge a conclu que la décision du maire constituait une voie de fait qui relevait du juge judiciaire. La nécessité de recourir aux tribunaux administratifs retarde évidemment l'implantation de lieux de cultes jéhovistes jusqu'à la conclusion de l'affaire en justice. Toutefois, elle ne semble pas l'empêcher. Le cas le plus évident est celui de la construction du Bethel de Louviers a été retardée mais

a quand même abouti. Le même phénomène s'est produit à Patterson (États-Unis) (Blanchard, 2003).

Seules, les modalités de résolution du problème diffèrent.

Les Témoins se sont aussi heurtés à la question des impôts fonciers. Quand ils possédaient une salle du Royaume, ils devaient acquitter en plus de la taxe d'habitation, l'impôt foncier puisqu'il ne sont pas reconnus comme association culturelle de droit plénier (lois de 1905 et de 1907). L'impôt foncier est une charge supplémentaire. Toutefois, les Témoins ont obtenu en justice l'exonération de ces taxes au même titre que les Églises reconnues. Ils n’acquittent plus la taxe d’habitation depuis 1993. Le Conseil d'État les a exonérés de la taxe foncière dans son arrêté du 23 juin 2000.

\section{Un mouvement messianique parmi d'autres}

Il n'existe pas de programme national de construction des temples jéhovistes mais en se dotant d'une association pour le développement des lieux des cultes (qui peut contracter un prêt à la construction) et en assurant les bâtiments avec un contrat collectif (Plan de protection des salles de Royaume), la Fédération chrétienne des Témoins de Jéhovah montre qu'elle ne se désintéresse pas des édifices cultuels qui appartiennent aux congrégations locales. Dans cette étude, nous nous attendions à pouvoir mettre en évidence plusieurs facteurs déterminant la construction des salles du royaume. Nous n'avons trouvé qu'un lien entre la croissance du nombre de salles du Royaume, le nombre de fidèles et le nombre de congrégations. Ceci est révélateur d'un mode de développement foncier que l'on ne trouve pas dans d'autres mouvements religieux. 
Il apparaît que la décision d'édifier son propre lieu de culte est une affaire locale qui se répète selon un même processus dans toutes les régions sans concertation nationale. Cela correspond donc à une tendance qui privilégie l’insertion durable dans la société (modèle dénominationel) à l'indifférence envers le temporel au profit de l'attente eschatologique (modèle sectaire). L’absence de lien mécanique entre la croissance du nombre de congrégations, le nombre de lieux de culte et l'émigration, invite à analyser les milieux dans lesquels le jéhovisme recrute actuellement ses nouveaux fidèles. Est-il concurrencé par les diverses formes du protestantisme évangélique dans les milieux de l'émigration?

1.Un Témoin de Jéhovah est un proclamateur car il a le devoir d'annoncer la bonne parole à toute l'humanité.

2.Le nom de l'organisation jéhoviste est la Watchtower Tact and Bible Society.

$\underline{3 . N o u s ~ e m p l o y o n s ~ l ' e x p r e s s i o n ~ e ́ d i f i c e ~ c u l t u e l ~ a u ~ s e n s ~ c o m m u n ~ e t ~ n o n ~ a u ~ s e n s ~ j u r i d i q u e ~ e t ~}$ fiscal car la revendication des exemptions fiscales liées à la reconnaissance comme « édifice cultuel » est en débat devant les tribunaux et le Conseil d’État. Sur ce point, les Témoins obtiennent de plus en plus satisfaction.

4. On trouve le même type d'assistance à l'union des assemblées de Dieu de France où la Mission intérieure (Nord) consent des prêts pour la construction ou l'agrandissement des lieux de culte.

5.Nous prenons toujours la population des 18-75 ans. 